\title{
MOTION ANALYSIS OF ECHOCARDIOGRAMS USING A LOCAL-AFFINE, SPATIO-TEMPORAL MODEL
}

\author{
Michael Sühling, Muthuvel Arigovindan, Patrick Hunziker*, and Michael Unser \\ Swiss Federal Institute of Technology Lausanne \\ Biomedical Imaging Group, STI/IOA \\ CH-1015 Lausanne, Switzerland \\ e-mail: michael.suehling@epfl.ch,muthuvel.arigovindan@epfl.ch,michael.unser@epfl.ch
}

\begin{abstract}
We present a new method for estimating heart motion from two-dimensional (2D) echocardiographic sequences. It is inspired by the Lucas-Kanade algorithm for optical flow which estimates motion parameters over a sliding window. However, instead of assuming that the motion is constant within the analysis window, we consider a model that is locally affine and can account for typical heart motions such as dilation/contraction and shear. Another refinement is spatial adaptivity which is achieved by estimating displacement vectors at multiple scales and selecting the most promising fit. The affine parameters are estimated in the least squares sense using a separable spatial (resp., spatio-temporal) Bspline window. This particular choice is motivated by the fact that the B-splines are nearly isotropic (Gaussian-like) and that they satisfy a two-scale equation. We use this latter property to derive a wavelet-like algorithm that leads to a fast computation of B-spline-weighted inner products and moments at dyadic scales, which speeds up our method considerably.

We test the algorithm on synthetic and real ultrasound sequences and show that it compares favorably with other methods, such as Lucas-Kanade and Horn-Schunk.
\end{abstract}

\section{INTRODUCTION}

Echocardiography is an effective imaging modality that enables clinicians to study the shape, size, and dynamics of the heart. The features that have made it so commonly used are its noninvasiveness and ease of use. The analysis of ventricular motion, in particular, provides an efficient mean to evaluate the degree of ischemia and infarction [1], [2]. Several methods have been proposed to quantify heart motion from 2D echocardiograms among which optical flow

\footnotetext{
${ }^{*} \mathrm{P}$. Hunziker is with the Kantonsspital Basel, Petersgraben 5, CH-4031 Basel, e-mail: PHunziker@uhbs.ch.

This work was supported in part by the Swiss National Science Foundation under grant 3200-059517.99 and the Swiss Heart Foundation.
}

methods have led to promising results [3]. For example, Mailloux et al. [4] extended the optical flow algorithm of Horn and Schunk [5]. A very popular optical flow algorithm is the Lucas-Kanade method [6], which estimates the motion locally, assuming that motion is constant within a window. This method was applied successfully to ultrasound by Chunke et al. [7].

In this paper, we introduce a variant of the Lucas-Kanade method that is better adapted to our situation. The refinements that we propose are four-fold:

- Instead of assuming that the motion is locally constant, we use a local-affine-in-space model for the displacement field, which is better suited to the description of typical heart motion such as dilation/contraction and shear. The local affine parameters are potentially very interesting for diagnostic purposes because they may offer a way to distinguish between passive and active tissue.

- Instead of working at a fixed scale, we consider estimation windows of increasing sizes and develop a multiresolution strategy to improve the estimation of large motions and to reduce the sensitivity to noise.

- We introduce a B-spline weighting scheme that has important computational advantages for multi-scale processing. In particular, we develop a wavelet-like multiresolution implementation.

- We also consider the option of adding a temporal affine component to our motion model. This leads to a more robust motion estimation which combines the information from multiple frames. It also yields an additional acceleration parameter-a useful indicator of heart dynamics.

The paper is organized as follows. We describe our method in some detail in Section 2. We then validate the algorithm in Section 3 by applying it to synthetic data that simulates the characteristics of a beating heart, and by comparing it 


$$
\begin{aligned}
& \mathbf{A}=\left(\begin{array}{cccccccc}
\left\langle w, I_{x}^{2}\right\rangle & \left\langle w, I_{x} I_{y}\right\rangle & \left\langle w, x I_{x}^{2}\right\rangle & \left\langle w, y I_{x}^{2}\right\rangle & \left\langle w, t I_{x}^{2}\right\rangle & \left\langle w, x I_{x} I_{y}\right\rangle & \left\langle w, y I_{x} I_{y}\right\rangle & \left\langle w, t I_{x} I_{y}\right\rangle \\
\cdot & \left\langle w, I_{y}^{2}\right\rangle & \left\langle w, x I_{x} I_{y}\right\rangle & \left\langle w, y I_{x} I_{y}\right\rangle & \left\langle w, t I_{x} I_{y}\right\rangle & \left\langle w, x I_{y}^{2}\right\rangle & \left\langle w, y I_{y}^{2}\right\rangle & \left\langle w, t I_{y}^{2}\right\rangle \\
\cdot & \cdot & \left\langle w, x^{2} I_{x}^{2}\right\rangle & \left\langle w, x y I_{x}^{2}\right\rangle & \left\langle w, x t I_{x}^{2}\right\rangle & \left\langle w, x^{2} I_{x} I_{y}\right\rangle & \left\langle w, x y I_{x} I_{y}\right\rangle & \left\langle w, x t I_{x} I_{y}\right\rangle \\
\cdot & \cdot & \cdot & \left\langle w, y^{2} I_{x}^{2}\right\rangle & \left\langle w, y t I_{x}^{2}\right\rangle & \left\langle w, x y I_{x} I_{y}\right\rangle & \left\langle w, y^{2} I_{x} I_{y}\right\rangle & \left\langle w, y t I_{x} I_{y}\right\rangle \\
& \cdot & \cdot & \cdot & \left\langle w, t^{2} I_{x}^{2}\right\rangle & \left\langle w, x t I_{x} I_{y}\right\rangle & \left\langle w, y t I_{x} I_{y}\right\rangle & \left\langle w, t^{2} I_{x} I_{y}\right\rangle \\
& \cdot & \cdot & \cdot & \left\langle w, x^{2} I_{y}^{2}\right\rangle & \left\langle w, x y I_{y}^{2}\right\rangle & \left\langle w, x t I_{y}^{2}\right\rangle \\
& & \cdot & \cdot & . & \left\langle w, y^{2} I_{y}^{2}\right\rangle & \left\langle w, y t I_{y}^{2}\right\rangle \\
& & & \cdot & . & . & \left\langle w, t^{2} I_{y}^{2}\right\rangle
\end{array}\right) \\
& \mathbf{v}=\left(u_{0}, v_{0}, u_{x}, u_{y}, u_{t}, v_{x}, v_{y}, v_{t}\right)^{T} \\
& \mathbf{b}=-\left(\left\langle w, I_{x} I_{t}\right\rangle,\left\langle w, I_{y} I_{t}\right\rangle,\left\langle w, x I_{x} I_{t}\right\rangle,\left\langle w, y I_{x} I_{t}\right\rangle,\left\langle w, t I_{x} I_{t}\right\rangle,\left\langle w, x I_{y} I_{t}\right\rangle,\left\langle w, y I_{y} I_{t}\right\rangle,\left\langle w, t I_{y} I_{t}\right\rangle\right)^{T}
\end{aligned}
$$

with alternative approaches. In particular, we test its robustness by adding various amounts of noise. Finally, we present some results with real echocardiographic data.

\section{LOCAL-AFFINE, MULTI-SCALE MOTION ESTIMATION}

Let $I(x, y, t)$ denote the intensity of pixels at location $\mathbf{r}=$ $(x, y)$ and time $t$ in an image sequence. Gradient-based optical flow estimation relies on the assumption that the intensity of a particular point in a moving pattern does not change with time. The constant intensity assumption can be expressed as [5]

$$
I_{x}(\mathbf{r}, t) u(\mathbf{r}, t)+I_{y}(\mathbf{r}, t) v(\mathbf{r}, t)+I_{t}(\mathbf{r}, t)=0
$$

where $I_{x}, I_{y}$ and $I_{t}$ denote the spatial and temporal derivatives of the image intensity. The velocities $u$ and $v$ are, respectively, the $x$ - and $y$-components of the optical flow we wish to estimate. Since (2) is a single equation in two unknown components $u$ and $v$, it cannot be solved uniquely without introducing additional constraints.

\subsection{Local Affine Velocity in Space-Time}

Let $\mathbf{r}_{0}=\left(x_{0}, y_{0}, t_{0}\right)$ denote the center of a small spatiotemporal image region $\Omega$. A very popular optical flow algorithm is the Lucas-Kanade method [6], which estimates the motion locally, assuming that motion is constant within a spatial window. In order to account for typical heart motions, such as expansion, contraction, and shear, we extend this approach to a local, spatial-affine model for the motion. Additionally, we also use a linear model for the velocity along the time direction. This allows to capture local accelerations in time much better than a locally constant model. Another advantage is that we can base our estimation on multiple frames around a given time point which is much more robust than using only two frames as many classical optical flow methods do. This spatio-temporal-affine model is defined as

$$
\begin{aligned}
& u(x, y, t)=u_{0}+u_{x}\left(x-x_{0}\right)+u_{y}\left(y-y_{0}\right)+u_{t}\left(t-t_{0}\right) \\
& v(x, y, t)=v_{0}+v_{x}\left(x-x_{0}\right)+v_{y}\left(y-y_{0}\right)+v_{t}\left(t-t_{0}\right)
\end{aligned}
$$

The parameters $u_{0}$ and $v_{0}$ correspond to the motion at the center point $\mathbf{r}_{0}$ and $u_{x}, u_{y}, u_{t}, v_{x}, v_{y}$, and $v_{t}$ are the first order spatial and temporal derivatives of $u$ and $v$, respectively.

We estimate the local motion components by minimizing the least-squares criterion

$$
\int_{\Omega} w(x, y, t)\left(I_{x} u+I_{y} v+I_{t}\right)^{2} d x d y d t .
$$

The symmetric window function $w$ gives more weight to constraints at the center of the local spatio-temporal region than to those at the periphery. A very well suited window function is $w(x, y, t)=\beta^{n}(x) \beta^{n}(y) \beta^{n}(t)$, where $\beta^{n}$ is the symmetrical B-spline of degree $n \in \mathbb{N}$ [8]. B-splines rapidly converge to Gaussians when their degree increases which ensures isotropy of the window in multiple dimensions. Additionally, B-splines satisfy a two-scale equation which allows for an efficient computation of B-spline-weighted inner products at dyadic scales by using a wavelet-like algorithm (cf. Section 2.2).

By differentiating (4) with respect to each of the eight unknown parameters, we obtain a symmetric linear system $\mathbf{A v}=\mathbf{b}$ in terms of local moments of the spatial and temporal derivatives of $I$, as defined in (1). More generally, the local moments at a given position $\mathbf{r}_{0}$ are given by

$$
\begin{array}{r}
m_{p, q, r}\left(x_{0}, y_{0}, t_{0}\right)=\int_{\Omega} w\left(x-x_{0}, y-y_{0}, t-t_{0}\right) \times \\
\left(x-x_{0}\right)^{p}\left(y-y_{0}\right)^{q}\left(t-t_{0}\right)^{r} f(x, y, t) d x d y d t
\end{array}
$$

where $0 \leq p+q+r \leq 2$ and $f$ represents the functions $I_{x}^{2}, I_{y}^{2}, I_{x} I_{y}, I_{x} I_{t}$, or $I_{y} I_{t}$. 


\subsection{Coarse-To-Fine Multi-Scale Strategy}

It is obviously difficult to estimate large motions at fine scales. A way around this problem is to apply a coarseto-fine strategy. At each spatial scale $j$ we use dilated and shifted versions of the window function:

$$
\begin{aligned}
& w^{(j)}\left(x-x_{0}, y-y_{0}, t-t_{0}\right)= \\
& w\left(\frac{x-2^{j} x_{0}}{2^{j}}, \frac{y-2^{j} y_{0}}{2^{j}}, t-t_{0}\right) .
\end{aligned}
$$

Note that the window functions at scale $j$ are spaced at a distance of $2^{j}$ pixels in the corresponding dimension. Computing inner products with large windows is computationally very expensive; however, B-spline functions satisfy a two-scale equation so that the local moments (5) can be computed recursively in a multiresolution approach using a Mallat-like algorithm [9]:

$$
\begin{array}{r}
m_{p, q, r}^{(j+1)}\left(x_{0}, y_{0}, t_{0}\right)= \\
\sum_{k=0}^{p} \sum_{l=0}^{q}\left(h_{p, k} h_{q, l} * m_{k, l, r}^{(j)}\right)\left(2 x_{0}, 2 y_{0}, t_{0}\right) .
\end{array}
$$

The one-dimensional two-scale filters $h_{i, j}$ depend on the degree of the chosen B-spline window and are applied separately in the $\mathrm{x}$ - and $\mathrm{y}$-directions. A more detailed description of this algorithm will be given elsewhere.

The motion vectors are cascaded through each resolution level as initial estimates and are then refined if they do not already exceed a scale-dependent size. For each local estimate, we compute a confidence measure which is based on the magnitude of the residual in (4). A local estimate is refined only if its confidence measure is larger than the corresponding one at the next coarser scale. Furthermore, a solution of a local linear system is regarded as not admissible if the linear system is either ill-conditioned or if the length of the estimated central motion vector exceeds some scale-dependent limit. Finally, a motion estimate is set to zero if the local mean of the time derivative at the given location is below a pre-defined noise level.

\section{NUMERICAL RESULTS}

For validation purposes, the algorithm was applied to synthetic and real sequences and compared to other methods. In order to obtain accurate numerical derivative estimates, we used cubic spline interpolation [8] in space and time.

\subsection{Application to Synthetic Data}

A quantitative analysis of the performance of the algorithm can only be done on synthetic sequences since the exact motion field is not known otherwise. In this study, the myocardium is modelled by a spherical ring containing a pat- (a) Frame during Expansion

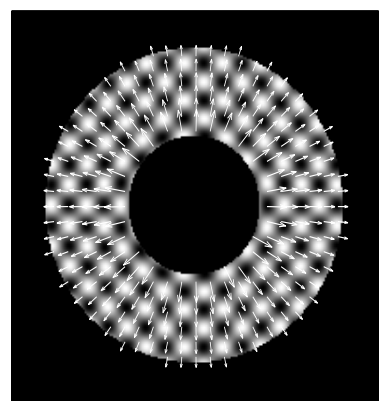

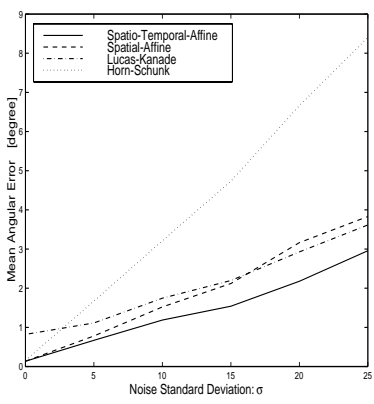

(b) Mean Angular Errors of different Methods
Fig. 1. One frame of the synthetic sequence and mean angular errors of the estimated motion fields.

tern representing tissue. To the inner layer of the ring (endocardium), we applied a cosine modulated velocity field in order to simulate expansion and contraction. The remaining myocardium was moving such that its area remains constant. This results in a radial velocity field with a magnitude decreasing with the distance from the center. This kind of motion reflects the wall-thinning and thickening of a beating heart during diastole and systole. One frame of the sequence together with its exact motion field is shown in Figure 1(a). The proposed algorithm was compared with two adaptations of the proposed approach. The first one only uses a local affine motion model in space and no window in the time dimension. The second adaptation corresponds to the LucasKanade method, which assumes the motion to be locally constant in space. The same spatial window and multiresolution strategy were used for these adaptations. Furthermore, we compared results with the method of Horn and Schunk. In particular, we used the regularization parameter $\alpha=3.0$, as recommended in [3], and performed at most 100 steps of the Gauss-Seidel iteration.

To test the robustness of the algorithms, we added gaussian white noise of different standard deviations to the original sequence. As in the real case, the noisy sequences were then prefiltered with a spatio-temporal Binomial filter of spatial variance $\sigma_{s}^{2}=1.0$ and temporal variance $\sigma_{t}^{2}=0.25$.

The error of the estimated motion fields was computed using the angular error measure as defined in [10]. The resulting mean angular errors of the different methods are illustrated in Figure 1(b). The worst method in the noise-free case is the Lucas-Kanade method. This is due to the fact that the diverging character of the motion field is not compatible with the local constancy-assumption. It starts behaving better as soon as the noise level increases, eventually even outperforming the affine-in-space method, which is less robust for high levels of noise simply because it has more pa- 


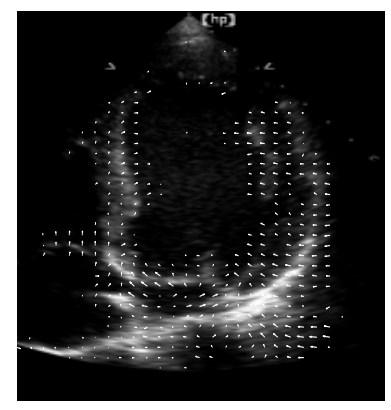

(a) Spatio-Temporal-Affine Method

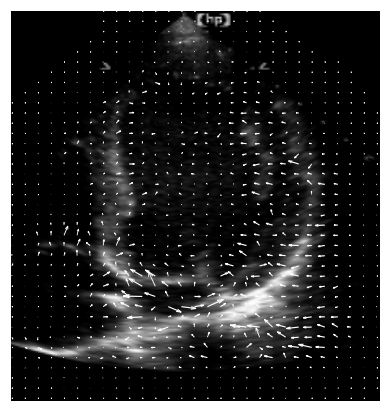

(b) Horn-Schunk Method

Fig. 2. One frame of the ultrasound sequence and the corresponding estimated motion fields. (Data curtesy of M. Sühling)

rameters to fit. The method of Horn and Schunk is clearly most sensitive to noise. Even by using a larger regularization parameter $\alpha=10.0$, the error did not decrease significantly. The spatio-temporal-affine algorithm performs best since the use of a time window provides increased robustness against noise.

\subsection{Application to Clinical Data}

For a first in vivo validation, we acquired 2D echocardiographic sequences by free-hand scanning using existing Hewlett-Packard hardware (HP Sonos 5500). Figure 2(a) shows one frame of an apical two-chamber-view sequence of a heart during systole (contraction). The corresponding estimated motion field using the spatio-temporal-affine method is superimposed in the form of a needle diagram. Figure 2(b) illustrates the corresponding motion field estimated by the method of Horn and Schunk with a value of $\alpha$ adapted for best subjective performance. Both motion fields clearly reflect the contraction of the myocardium. However, the results of the Horn-Schunk method suffer from two artifacts: 1) a less coherent motion field (e.g. more noise) in the moving areas of the heart, and 2) a significant smoothing of the local motion across the boundaries of the myocardium which comes as the consequence of the use of a global regularization.

\section{CONCLUSION}

We proposed a new method to estimate heart motion from echocardiographic sequences which uses a spatio-temporalaffine model for the velocity within a local window. We also introduced a nearly isotropic weighting scheme which uses B-splines. A hierarchical refinement scheme using a

dyadic scale progression was applied to reduce the problems of estimating large motions and to reduce the sensitivity to noise. Computational efficiency was achieved by developing a Mallat-like algorithm for computing B-splineweighted inner products and moments at dyadic scales. Accuracy and noise robustness of the proposed method was demonstrated on synthetic and real ultrasound sequences.

\section{REFERENCES}

[1] P. R. Hunziker, M. H. Picard, N. Jander, M. ScherrerCrosbie, M. Pfisterer, and P. T. Buser, "Regional wall motion assessment in stress echocardiography by tissue doppler bull's-eyes.," Journal of the American Society of Echocardiography, vol. 12, no. 3, pp. 196202, March 1999.

[2] X. Papademetris and J. S. Duncan, Handbook of Medical Imaging, vol. 2, chapter 12: Cardiac Image Analysis: Motion and Deformation, SPIE PRESS, 2000.

[3] P. Baraldi, A. Sarti, C. Lamberti, A. Prandini, and F. Sgallari, "Evaluation of differential optical flow techniques on synthesized echo images," IEEE Transactions on Biomedical Engineering, vol. 43, no. 3, pp. 259-272, March 1996.

[4] G. E. Mailloux, F. Langlois, P. Y. Simard, and M. Bertrand, "Restoration of the velocity field of the heart from two-dimensional echocardiograms," IEEE Transactions on Medical Imaging, vol. 8, no. 2, pp. 143-153, June 1989.

[5] B. Horn and B. Schunk, "Determining optical flow," Artificial Intelligence, vol. 17, pp. 185-203, 1981.

[6] B. Lucas and T. Kanade, "An iterative image restoration technique with an application to stereo vision," Proceedings of the DARPA IU Workshop, pp. 121130, 1981.

[7] Y. Chunke, K. Terada, and S. Oe, "Motion analysis of echocardiograph using optical flow method," IEEE International Conference on Systems, Man and Cybernetics, vol. 1, pp. 672-677, 1996.

[8] M. Unser, "Splines: A perfect fit for signal and image processing," IEEE Signal Processing Magazine, vol. 16, no. 6, pp. 22-38, November 1999.

[9] S. Mallat, "Multiresolution approximation and wavelets," Trans. Amer. Math. Soc., vol. 315, pp. 6988, 1989.

[10] J. Barron, D. Fleet, S. Beauchemin, and T. Burkitt, "Performance of optical flow techniques," International Journal of Computer Vision, vol. 12, pp. 43-77, 1994. 